\title{
Bacterial diversity of the American sand fly Lutzomyia intermedia using high-throughput metagenomic sequencing
}

\author{
Carolina Cunha Monteiro', Luis Eduardo Martinez Villegas', Thais Bonifácio Campolina', \\ Ana Clara Machado Araújo Pires ${ }^{1}$, Jose Carlos Miranda², Paulo Filemon Paolucci Pimenta ${ }^{1}$ and \\ Nagila Francinete Costa Secundino ${ }^{1 *}$
}

\begin{abstract}
Background: Parasites of the genus Leishmania cause a broad spectrum of diseases, collectively known as leishmaniasis, in humans worldwide. American cutaneous leishmaniasis is a neglected disease transmitted by sand fly vectors including Lutzomyia intermedia, a proven vector. The female sand fly can acquire or deliver Leishmania spp. parasites while feeding on a blood meal, which is required for nutrition, egg development and survival. The microbiota composition and abundance varies by food source, life stages and physiological conditions. The sand fly microbiota can affect parasite life-cycle in the vector.

Methods: We performed a metagenomic analysis for microbiota composition and abundance in Lu. intermedia, from an endemic area in Brazil. The adult insects were collected using CDC light traps, morphologically identified, carefully sterilized, dissected under a microscope and the females separated into groups according to their physiological condition: (i) absence of blood meal (unfed = UN); (ii) presence of blood meal (blood-fed = BF); and (iii) presence of developed ovaries (gravid=GR). Then, they were processed for metagenomics with Illumina Hiseq Sequencing in order to be sequence analyzed and to obtain the taxonomic profiles of the microbiota.

Results: Bacterial metagenomic analysis revealed differences in microbiota composition based upon the distinct physiological stages of the adult insect. Sequence identification revealed two phyla (Proteobacteria and Actinobacteria), 11 families and 15 genera; $87 \%$ of the bacteria were Gram-negative, while only one family and two genera were identified as Gram-positive. The genera Ochrobactrum, Bradyrhizobium and Pseudomonas were found across all of the groups.

Conclusions: The metagenomic analysis revealed that the microbiota of the Lu. intermedia female sand flies are distinct under specific physiological conditions and consist of 15 bacterial genera. The Ochrobactrum, Bradyrhizobium and Pseudomonas were the common genera. Our results detailing the constituents of Lu. intermedia native microbiota contribute to the knowledge regarding the bacterial community in an important sand fly vector and allow for further studies to better understand how the microbiota interacts with vectors of human parasites and to develop tools for biological control.
\end{abstract}

Keywords: Lutzomyia intermedia, Native microbiota, Metagenomics

Abbreviations: ACL, American cutaneous leishmaniasis; BF, Blood-fed; GR, Gravid; OTUs, Operational taxonomic units (OTUs); QIIME, Quantitative insights into microbial ecology; RDP, Ribosomal database project; UN, Unfed

\footnotetext{
*Correspondence: nagila@cpqrr.fiocruz.br

'Laboratory of Medical Entomology, René Rachou Research Centre

(FIOCRUZ-MG), Belo Horizonte, Minas Gerais, Brazil

Full list of author information is available at the end of the article
} 


\section{Background}

Parasites of the genus Leishmania cause a broad spectrum of diseases in humans worldwide, collectively known as leishmaniasis. American cutaneous leishmaniasis (ACL) is a neglected disease found in 18 countries and approximately 60,000 new cases are diagnosed every year [1]. In Brazil, it is present in all states [2] and is prevalent mainly in the northern and northeastern regions. Even though several Leishmania spp. have been described, Leishmania braziliensis is the main species causative of ACL and can be transmitted by different sand fly species in distinct endemic geographical regions. In fact, there are several sand fly species recognized as proven vectors of $L$. braziliensis, including Lutzomyia intermedia, Lutzomyia whitmani, Lutzomyia neivai, Lutzomyia migonei, Lutzomyia wellcomei and Lutzomyia complexa [3].

In the southeastern region of Brazil, Lutzomyia intermedia is the main vector of $L$. braziliensis $[4,5]$. This opportunistic species is associated with forests, nature reserves and modified domestic and peridomestic environments, such as animal shelters [6-8].

The female sand fly can acquire or deliver Leishmania spp. parasites while feeding on a blood meal, which is required for nutrition, egg development and survival. All events involved in parasite transformation, development, survival and establishment of vector infection occur in the digestive tract. A variety of microorganisms, such as fungi and bacteria, can interact with Leishmania spp. in the vector and during their transmission to a vertebrate host. Bacterial communities have been studied to understand how bacteria interact with parasites. The insect microbiota varies by food source and life stage; during the immature stage, sand fly larvae ingest microorganisms from the soil environment. Furthermore, adult sand flies have the opportunity to ingest microorganisms, either from plant sap or sticky honeydew from aphids. Alternatively, adult females also ingest yeasts and bacteria from their vertebrate hosts used as blood source [9-13]. All these microbes will contribute to form their midgut microbiota.

In 2002, Volf et al. [10] studying $P$. duboscqi showed microbiota modulation due to blood meal. Two days after the ingestion of a blood meal, there was an increase in bacterial number in the midgut followed by a decrease after blood meal digestion. This fact is in line with other studies performed with other nematoceran insects (i.e. Aedes aegypti and Anopheles gambiae) showing an increase in their midgut microbiota after a blood meal [14, 15]. More recently, in vitro effect of microbiota on Leishmania spp. survival was demonstrated showing that Serratia, Bacillus and Haemophilus parainfluenzae induced lysis of promastigote forms [12, 16, 17]. Moreover, sand flies pre-fed with Pseudozyma, Asaia or Ochrobactrum showed a reduction in parasite survival [18].
Here, we performed a metagenomic analysis for microbiota composition and abundance in female adult sand flies, specifically in Lu. intermedia originated from an endemic area in Brazil. This study establishes basic knowledge for future studies to understand the role of microbiota during the course of $L$. braziliensis infection in this important vector.

\section{Methods \\ Sand flies}

Lutzomyia intermedia adults were collected using CDC light traps [19] from a locality in Corte de Pedra, State of Bahia, Brazil (longitude $59^{\circ} 30^{\prime} \mathrm{W}$; latitude $13^{\circ} 26^{\prime} \mathrm{S}$ ); a hilly region (600 $\mathrm{m}$ above sea level) covered by different stages of secondary Atlantic forests. Corte de Pedra is a village with human interference involved with cocoa and clove plantations. The light traps were placed around animal shelter in April 2014.

\section{Specimen preparation}

The collected sand flies were anesthetized at $-20{ }^{\circ} \mathrm{C}$ for a few seconds and had their surfaces carefully sterilized to prepare for serial passage as follows: $10 \mathrm{~s}$ in $70 \%$ ethanol, $1 \mathrm{~min}$ in $1 \%$ hypochlorite and 1-min wash in PBS three times [20]. The specimens were morphologically identified according to Young \& Duncan [21]. Sand fly midguts were dissected under a stereoscope and separated into groups according to their physiological condition: (i) absence of blood meal (unfed $=\mathrm{UN}$ ); (ii) presence of blood meal (blood-fed = BF); and (iii) presence of developed ovaries (gravid =GR). The pooled samples (300 of each group) were stored in micro centrifuge tubes with $(100 \mu \mathrm{l})$ of RNAlater ${ }^{\circ}$ (Qiagen, Hilden, GER) at $4{ }^{\circ} \mathrm{C}$ for later RNA extraction and metagenomic analysis.

\section{Metagenomic process RNA extraction}

Samples previously stored in RNAlater were washed in distilled water using a $35 \mu \mathrm{m}$ nylon filter prior to RNA extraction. RNA extraction was performed in two steps. First, the pooled samples were ground in TRI Reagent ${ }^{\oplus}$ RT (Molecular Research Center, Inc., Cincinnati, USA) and chloroform with Teflon pestle and then incubated at room temperature for $15 \mathrm{~min}$. The samples were centrifuged at $15,294 \mathrm{rcf}$ at $4{ }^{\circ} \mathrm{C}$ for $15 \mathrm{~min}$ followed by the addition of $70 \%$ ethanol to the supernatant. The supernatant was transferred to a column (RNeasy Mini Kit, Qiagen, Hilden, GER) and processed according to the manufacturer's protocol. RNA quality was assessed using a NanoDrop spectrophotometer (Thermo Fisher Scientific, Massachusetts, USA), and $50 \mathrm{ng} / \mu \mathrm{l}$ of RNA was used to synthetize cDNA using the Reverse Transcription Kit (Qiagen, Hilden, GER). cDNA was diluted $10 \times$, stored at $-20{ }^{\circ} \mathrm{C}$ and submitted for high-throughput Illumina sequencing. 


\section{Illumina HiSeq sequencing}

cDNA from the three sand fly experimental groups were sent to the DNA Service Platform (University of Illinois, USA), where the samples were sequenced (http:// www.biotech.illinois.edu/htdna/services-equipment). The $16 \mathrm{~S}$ ribosomal cDNA primers used were the following: CS1_27F sense: 5'-ACA CTG ACG ACA TGG TTC TAC AAG AGT TTG ATC CTG GCT CAG CS2_534R and antisense: 5'-TAC GGT AGC AGA GAC TTG GTC TAT TAC CGC GGC TGC TGG-3' (http://hmpdacc.org).

\section{Sequence analysis and taxonomic profiling}

The reads corresponding to the targeted 16S rRNA V1-V3 region were $\mathrm{QC}$ processed using the tools implemented within the Quantitative Insights Into Microbial Ecology (QIIME) software v1.7.0 pipeline [22]. Briefly, primer sequences were removed, and reads were trimmed at the extremities using a Phred value Q20. High quality reads ( $\geq 250 \mathrm{bp}$ ) were further processed to remove chimera sequences using ChimeraSlayer. Sequences within an identity threshold of $97 \%$ were clustered into Operational Taxonomic Units (OTUs) using the UCLUST [23] pipeline. The most abundant sequence within each of the clusters was selected as representative, and its taxonomic affiliation was assigned using the Ribosomal Database Project (RDP) classifier [24] via QIIME considering a minimum confidence value of $80 \%$.

The bacterial community composition of each experimental group was compiled considering only OTUs encompassing at least a $0.5 \%$ relative abundance. The cutoff value was set to address rare sequences that most likely corresponded to random sequencing errors (eg, previously reported thresholds for community composition tables in other insect models vary from 1 to $0.04 \%[25,26]$.

\section{Results}

High-throughput Illumina sequencing

Sequencing revealed a total of 587,144 valid reads, which were grouped as follows: UN: 117,492; GR: 44,318; and BF 425,334. After the sequences were quality checked and assembled, the UN group was clustered into 66 OTUs, the GR group into 30 OTUs, and the BF group into 40 OTUs; only 12, 9 and 8 OTUs in the UN, GR and $\mathrm{BF}$ groups respectively, were considered valid bacterial OTUs according to the cut-off value. An average of $2 \%$ reads were unassigned and $2.9,1.4$, and $1.4 \%$ of OTUs fell below the cut-off line in the UN and GR and $\mathrm{BF}$ groups, respectively.

\section{Diversity of bacterial microbiota}

Bacterial metagenomic analysis revealed differences in microbiota composition based upon the distinct physiological stages of the adult insect. Sequence identification revealed two phyla (Proteobacteria and Actinobacteria),
11 families and 15 genera; $87 \%$ of the bacteria were Gram-negative, while only one family and two genera were identified as Gram-positive (Table 1).

\section{Correlation in diversity and abundance}

Lutzomyia intermedia microbiota diversity and abundance varied based on the studied groups. In the UN and GR groups, the family Enterobacteriaceae was the most abundant, representing $54 \%$ and $47 \%$ of each group, respectively. Interestingly, in the $\mathrm{BF}$ group, the family Rickettsiaceae was the most prevalent, with $46.7 \%$ of the group represented by Wolbachia and only $4.3 \%$ of the group represented by Enterobacteriaceae. The genera Ochrobactrum, Bradyrhizobium and Pseudomonas were found across all of the groups.

The abundance of the genera Bradyrhizobium and Pseudomonas increased after the blood meal (6.0-fold and 3.8-fold, respectively), but abundance increased more in gravid sand flies (8.2-fold and 18.0-fold, respectively). Particularly, the abundance of Ochrobactrum bacteria decreased 2.2-fold and 3.7-fold in the BF and GR sand fly groups, respectively (Fig. 1).

\section{Discussion}

Knowledge of the microbiota in the vectors of humanborne diseases is essential to comprehend the relationships with the pathogens they transmit. To our knowledge, this is the first investigation of the native microbiota of $L u$. intermedia, a proven vector of $L$. braziliensis. Sand flies were collected near Corte de

Table 1 Taxonomic classification of the metagenomic OTUs of Lutzomyia intermedia

\begin{tabular}{ll}
\hline Family & Genus (Gram stain) \\
\hline Bradyrhizobiaceae & Bradyrhizobium (-) \\
Brucellaceae & Ochrobactrum $(-)$ \\
Comamonadaceae & Pelomonas $(-)$ \\
Enterobacteriaceae & Enterobacter $(-)$ \\
& Erwinia $(-)$ \\
& Kluyvera $(-)$ \\
Methylobacteriaceae & Serratia $(-)$ \\
Moraxellaceae & Unidentified genus \\
Neisseriaceae & Unidentified genus \\
Propionibacteriaceae & Acinetobacter $(-)$ \\
& Kingella $(-)$ \\
Pseudomonadaceae & Curtobacterium $(+)$ \\
Ralstoniaceae & Propionibacterium $(+)$ \\
Rickettsiaceae & Pseudomonas $(-)$ \\
& Ralstonia $(-)$ \\
\hline & Rickettsia $(-)$ \\
& Wolbachia $(-)$ \\
\hline
\end{tabular}




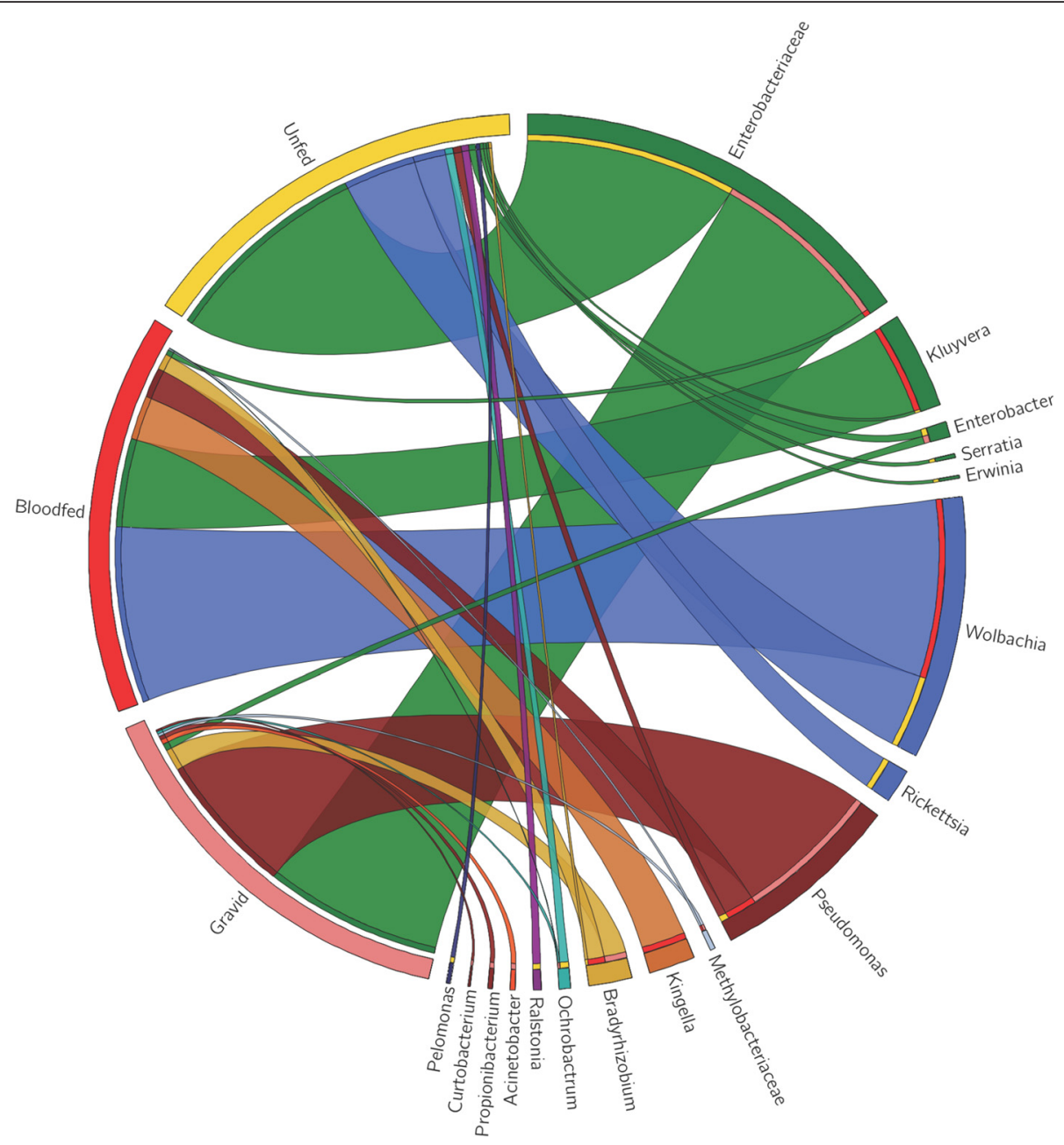

Fig. 1 The circular web represents the relative abundance of the bacterial components of Lutzomyia intermedia microbiota identified by New Generation Sequencing. Generated with Circos online tool [39]

Pedra, a small village and cutaneous leishmaniasis endemic area where 2,384 new cases of ACL were registered in 2015 [27]. Metagenomic analysis revealed only two phyla: (i) Proteobacteria, a dominant phylum with ten families and 13 genera, and (ii) Actinobacteria, with only one family and two genera.

The majority of these families originate from the environment [28-30]. Sand flies can acquire several species of bacteria from the soil during larva nurturing and in plant sap during adult feeding [10-13]. The most abundant family in the UN and GR groups was the Enterobacteriaceae, while the Rickettsiaceae was the most prevalent in the BF group. The Enterobacteriaceae is described as the most common family present in insects. Although the majority of the data was obtained from the UN and GR groups (64,917 and 20,809 reads, respectively), it was not possible to identify the Enterobacteriaceae members into genera taxon due to the similarity of the $16 \mathrm{~S}$ rRNA variable regions [31]. In fact, these data are supported by other studies that detected members of Enterobacteriaceae in New and Old World sand flies using cultivation-dependent techniques [9, 32-34].

Three genera were present in Lu. intermedia in all experimental groups: Ochrobactrum, Bradyrhizobium and Pseudomonas. This is the first finding of Ochrobactrum in wild New World sand flies, as it was previously reported only in P. duboscqi, an Old World leishmaniasis vector and in $\mathrm{Lu}$. longipalpis [10, 18]. Similar to our study, Bradyrhizobium was described in Lu. longipalpis and Lutzomyia cruzi, both New World leishmaniasis vectors [35]. Pseudomonas was found in both genera, Phlebotomus and Lutzomyia, from Old and New World sand flies, including $L u$. longipalpis [11, 31, 32, 36], P. argentipes, $P$. duboscqi, $P$. papatasi, $P$. perfiliewi and $P$. sargenti $[9,10,13,33,37,38]$.

We assumed that the microbiota from the midgut of unfed $\mathrm{Lu}$. intermedia sand flies is a quiescent community of bacteria. These sand flies had no previous contact 
with blood meal; as such, this group was considered a control group and was used to proportionally compare the abundance of bacterial genera present in the other groups. Fed sand flies showed a 33.4-fold and 2.45-fold increase in the abundance of Kluyvera and Wolbachia, respectively, compared to controls due to the presence of blood meal in the midgut. Moreover, the abundance of the genera Bradyrhizobium and Pseudomonas also increased after the blood meal (6.0-fold and 3.8-fold, respectively), but the abundance increased more in gravid sand flies (8.2-fold and 18.0-fold, respectively).

Particularly, the abundance of Ochrobactrum bacteria decreased 2.2-fold and 3.7-fold in the blood-fed and gravid sand flies, respectively. Curiously, the Enterobacter present in the unfed sand flies were not detected in the blood-fed sand flies but were present at the same abundance in the gravid sand flies. It is interesting to observe that Kluyvera and Pseudomonas, both found in soil and plants, and Wolbachia, a maternally transmitted endosymbiont, are the microbiota genera that were most enhanced by the presence of blood meal in the midgut.

The family Enterobacteriacea remained present as the second most abundant after the blood meal represented by the genus Kluyvera. In our opinion, this is expected since this family is favored in hematophagous insects after the blood meal. In 2011, Wang et al. [15] showed that members of Enterobacteriacea proliferate under oxidative stress due to their capacity to cope with the oxidative stress. Interestingly, Wolbachia spp. were most predominant. Wolbachia spp. are intracellular organisms and for this reason are protected from the highly oxidative environment in the gut lumen. Until today, the nature of the relation between $\mathrm{Wol}$ bachia spp. and sand flies is still obscure.

\section{Conclusion}

Our metagenomic analysis revealed that the microbiota of female $\mathrm{Lu}$. intermedia sand flies are distinct under specific physiological conditions and consist of 15 bacterial genera with Ochrobactrum, Bradyrhizobium and Pseudomonas being the common genera. We demonstrated that the presence of a blood meal influences bacterial abundance in the midgut, in a more exacerbated way than the gravid condition. Our results detailing the constituents of $L u$. intermedia native microbiota contribute to the knowledge of bacterial communities in an important sand fly vector and allow for further studies to better understand how the microbiota interacts with vectors of human parasites and to develop tools for biological control.

\section{Acknowledgments}

This study was partially funded by the following Brazilian agencies: Foundation of the Institute Oswaldo Cruz (FIOCRUZ), Brazilian Council for Scientific and Technological Development (CNPq), Minas Gerais State Research Support Foundation (FAPEMIG). Thanks to the Elsevier Language Editing Services.
Funding

NFCS and PFPP are research fellows, supported by CNPq. CCM, TBC and ACMAP are students, supported by CAPES and LEMV is supported by CNPq.

Availability of data and materials

The dataset generated during the current study are available from the corresponding author upon a reasonable request.

\section{Authors' contributions}

Conceived and designed the experiments: NFCS, PFPP, CCM. Performed the experiments: CCM, JCM, TBC, ACMAP. Analyzed the data: NFCS, CCM, LEMV. Wrote the paper: NFCS, PFPP CCM. All authors read and approved the final version of the manuscript.

\section{Competing interests}

The authors declare that they have no competing interests.

Consent for publication

Not applicable.

\section{Ethics approval and consent to participate}

This study was conducted in accordance with the recommendations from the Manual for the Use of Animals/FIOCRUZ, Ministry of Health of Brazil (National decree $\mathrm{Nr} 3$,179). The protocol was approved by the Ethics Committee for the Use of Animals of the Fundação Oswaldo Cruz - FIOCRUZ, Ministry of Health of Brazil (Nr L-1715).

\section{Author details}

${ }^{1}$ Laboratory of Medical Entomology, René Rachou Research Centre (FIOCRUZ-MG), Belo Horizonte, Minas Gerais, Brazil. ${ }^{2}$ Centro de Pesquisas Gonçalo Moniz (CPqGM)-Fundação Oswaldo Cruz (FIOCRUZ), Salvador, Bahia, Brazil.

Received: 4 May 2016 Accepted: 19 August 2016

Published online: 31 August 2016

References

1. Pan American Health Organization. Epidemiological report of the Americas. Report leishmaniases. 2015.

2. Ministerio da Saúde, Secretaria de Vigilância em Saúde: Manual de recomendações para diagnóstico, tratamento e acompanhamento de pacientes com a coinfecção leishmania-HIV / Ministério da Saúde, Secretaria de Vigilância em Saúde, Departamento de Vigilância das Doenças Transmissíveis. 2015.

3. Brazil RP, Rodrigues AAF, Filho JDA. Sand Fly vectors of leishmania in the Americas - a mini review. Entomol Ornithol Herpetol. 2015;4:144.

4. Rangel EF, Lainson R. Importancia medico-veterinaria. In: Rangel EF, Lainson R, editors. Flebotomineos do Brasil. Rio de Janeiro: Fiocruz; 2003. p. 15-9.

5. de Brito M, Casanova C, Mascarini LM, Wanderley DM, Correa FM. Phlebotominae (Diptera: Psychodidae) in area of transmission of american tegumentar leishmaniasis in the north coast of the State of Sao Paulo, Brazi. Rev Soc Bras Med Trop. 2002;35 Suppl 5:431-7.

6. Forattini OP. Psychodidae, Leishmanioses, Bartenolose. In: Blucher E, editor Entomologia Medica. São Paulo: Edgard Blucher/Edusp; 1973.

7. Miranda JC, Reis E, Schriefer A, Goncalves M, Reis MG, Carvalho L, et al. Frequency of infection of Lutzomyia phlebotomines with Leishmania braziliensis in a Brazilian endemic area as assessed by pinpoint capture and polymerase chain reaction. Mem Inst Oswaldo Cruz. 2002;97 Suppl 2:185-8.

8. de Souza CF, Brazil RP, Bevilacqua PD, Andrade Filho JD. The phlebotomine sand flies fauna in Parque Estadual do Rio Doce, Minas Gerais, Brazil. Parasit Vectors. 2015;8:619.

9. Dillon RJ, el Kordy E, Shehata M, Lane RP. The prevalence of a microbiota in the digestive tract of Phlebotomus papatasi. Ann Trop Med Parasitol. 1996;90 Suppl 6:669-73.

10. Volf P, Kiewegova A, Nemec A. Bacterial colonisation in the gut of Phlebotomus duboseqi (Diptera: Psychodidae): transtadial passage and the role of female diet. Folia Parasitol (Praha). 2002;49 Suppl 1:73-7.

11. Peterkova-Koci K, Robles-Murguia M, Ramalho-Ortigao M, Zurek L. Significance of bacteria in oviposition and larval development of the sand fly Lutzomyia longipalpis. Parasit Vectors. 2012;5:145. 
12. Hassan Ml, Al-Sawaf BM, Fouda MA, Al-Hosry S, Hammad KM. A recent evaluation of the sandfly, Phlepotomus papatasi midgut symbiotic bacteria effect on the survivorship of Leishmania major. J Anc Dis Prev Rem. 2014;2 Suppl 1:110.

13. Maleki-Ravasan N, Oshaghi MA, Afshar D, Arandian MH, Hajikhani S, Akhavan AA, Yakhchali B, Shirazi MH, Rassi Y, Jafari R, et al. Aerobic bacterial flora of biotic and abiotic compartments of a hyperendemic Zoonotic Cutaneous Leishmaniasis (ZCL) focus. Parasit Vectors. 2015;8:63.

14. Oliveira JH, Goncalves RL, Lara FA, Dias FA, Gandara AC, Menna-Barreto RF, et al. Blood meal-derived heme decreases ROS levels in the midgut of Aedes aegypti and allows proliferation of intestinal microbiota. PLoS Pathog. 2011;7 Suppl 3, e1001320.

15. Wang S, Ghosh AK, Bongio N, Stebbings KA, Lampe DJ, Jacobs-Lorena M. Fighting malaria with engineered symbiotic bacteria from vector mosquitoes. Proc Natl Acad Sci U S A. 2012;109 Suppl 31:12734-9.

16. Moraes CS, Seabra SH, Castro DP, Brazil RP, de Souza W, Garcia ES, Azambuja P. Leishmania (Leishmania) chagasi interactions with Serratia marcescens: ultrastructural studies, lysis and carbohydrate effects. Exp Parasitol. 2008;118 Suppl 4:561-8.

17. Moraes CS, Seabra SH, Albuquerque-Cunha JM, Castro DP, Genta FA, de Souza W, et al. Prodigiosin is not a determinant factor in lysis of Leishmania (Viannia) braziliensis after interaction with Serratia marcescens D-mannose sensitive fimbriae. Exp Parasitol. 2009;122 Suppl 2:84-90.

18. Sant'Anna MR, Diaz-Albiter H, Aguiar-Martins K, Al Salem WS, Cavalcante RR, Dillon VM, et al. Colonisation resistance in the sand fly gut: Leishmania protects Lutzomyia longipalpis from bacterial infection. Parasit Vectors. 2014;7:329.

19. Sudia WD, Chamberlain RW. Battery-operated light trap, an improved model. By W. D. Sudia and R. W. Chamberlain, 1962. J Am Mosq Control Assoc. 1988;4 Suppl 4:536-8.

20. Lacey LA, Brooks WM. Initial handling and diagnosis of diseased insects. In: Lacey LA, editor. Manual of techniques in insect pathology. San Diego: Elsevier Science; 1997. p. 409

21. Young DG, Duncan MA. Guide to the idenfication and geographic distribution of Lutzomyia sand flies in Mexico, the West Indies, Central and South America. Mem Am Ent Soc. 1994;54:881.

22. Caporaso JG, Bittinger K, Bushman FD, DeSantis TZ, Andersen GL, Knight R. PYNAST: a flexible tool for aligning sequences to a template alignment. Bioinformatics. 2010:26 Suppl 2:266-7.

23. Edgar RC. Search and clustering orders of magnitude faster than BLAST. Bioinformatics. 2010;26 Suppl 19:2460-1.

24. Cole JR, Chai B, Farris RJ, Wang Q, Kulam SA, McGarrell DM, et al. The Ribosomal Database Project (RDP-II): sequences and tools for highthroughput rRNA analysis. Nucleic Acids Res. 2005;33:D294-6.

25. Wang Y, Gilbreath III TM, Kukutla P, Yan G, Xu J. Dynamic gut microbiome across life history of the malaria mosquito Anopheles gambiae in Kenya. PLoS One. 2011;6 Suppl 9, e24767.

26. Boissiere A, Tchioffo MT, Bachar D, Abate L, Marie A, Nsango SE, et al. Midgut microbiota of the malaria mosquito vector Anopheles gambiae and interactions with Plasmodium falciparum infection. PLoS Pathog. 2012;8 Suppl 5, e1002742.

27. SUVISA and Secretaria da Saude do Estado da Bahia: Vigilância em Saúde Proteção da Saúde. Portal da Vigilância.http://www.suvisa.saude.ba.gov.br/ informacao_saude. Accessed 1 Apr 2015.

28. Genin S, Boucher C. Ralstonia solanacearum: secrets of a major pathogen unveiled by analysis of its genome. Mol Plant Pathol. 2002;3 Suppl 3:111-8.

29. Kay E, Bertolla F, Vogel TM, Simonet P. Opportunistic colonization of Ralstonia solanacearum-infected plants by Acinetobacter sp. and its natural competence development. Microb Ecol. 2002;43 Suppl 3:291-7.

30. Angot A, Peeters N, Lechner E, Vailleau F, Baud C, Gentzbittel L, et al. Ralstonia solanacearum requires Fbox-like domain-containing type III effectors to promote disease on several host plants. Proc Natl Acad Sci U S A. 2006;103 Suppl 39:14620-5.

31. Chakravorty S, Helb D, Burday M, Connell N, Alland D. A detailed analysis of 165 ribosomal RNA gene segments for the diagnosis of pathogenic bacteria. J Microbiol Methods. 2007;69 Suppl 2:330-9.

32. de Oliveira SM P, de Morais BA, Goncalves CA, Giordano-Dias CM, Vilela ML, Brazil RP, et al. Digestive tract microbiota in female Lutzomyia longipalpis (Lutz \& Neiva, 1912) (Diptera: Psychodidae) from colonies feeding on blood meal and sucrose plus blood meal. Cad Saude Publica. 2001;17 Suppl 1:229-32.

33. Gouveia C, Asensi MD, Zahner V, Rangel EF, Oliveira SM. Study on the bacterial midgut microbiota associated to different Brazilian populations of
Lutzomyia longipalpis (Lutz \& Neiva) (Diptera: Psychodidae). Neotrop Entomol. 2008:37 Suppl 5:597-601.

34. Akhoundi M, Bakhtiari R, Guillard T, Baghaei A, Tolouei R, Sereno D, et al. Diversity of the bacterial and fungal microflora from the midgut and cuticle of phlebotomine sand flies collected in North-Western Iran. PLoS One. 2012;7 Suppl 11, e50259.

35. Sant'Anna MR, Darby AC, Brazil RP, Montoya-Lerma J, Dillon VM, Bates PA, Dillon RJ. Investigation of the bacterial communities associated with females of Lutzomyia sand fly species from South America. PLoS One. 2012;7 Suppl 8, e42531.

36. Oliveira SM, Moraes BA, Goncalves CA, Giordano-Dias CM, D'Almeida JM Asensi MD, et al. Prevalence of microbiota in the digestive tract of wild females of Lutzomyia longipalpis Lutz \& Neiva, 1912) (Diptera: Psychodidae). Rev Soc Bras Med Trop. 2000;33 Suppl 3:319-22.

37. Hillesland H, Read A, Subhadra B, Hurwitz I, McKelvey R, Ghosh K, et al. Identification of aerobic gut bacteria from the kala azar vector, Phlebotomus argentipes: a platform for potential paratransgenic manipulation of sand flies. Am J Trop Med Hyg. 2008;79 Suppl 6:881-6.

38. Maleki-Ravasan N, Oshaghi MA, Hajikhani S, Saeidi Z, Akhavan AA, GeramiShoar $M$, et al. Aerobic microbial community of insectary population of Phlebotomus papatasi. J Arthropod Borne Dis. 2014;8 Suppl 1:69-81.

39. Krzywinski M, Schein J, Birol I, Connors J, Gascoyne R, Horsman D, Jones SJ, Marra MA. Circos: an information aesthetic for comparative genomics. Genome Res. 2009:19 Suppl 9:1639-45.

\section{Submit your next manuscript to BioMed Central and we will help you at every step:}

- We accept pre-submission inquiries

- Our selector tool helps you to find the most relevant journal

- We provide round the clock customer support

- Convenient online submission

- Thorough peer review

- Inclusion in PubMed and all major indexing services

- Maximum visibility for your research

Submit your manuscript at www.biomedcentral.com/submit
) Biomed Central 